SEI,ECTION FOR TWINS IN BEEF HERDS

\author{
L. R. PIPER $\left({ }^{*}\right)$, B. M. BINDON $\left({ }^{*}\right)$ and R. BARLOW $\left(^{+}\right)$
}

(*) CSIRO, Division of Animal Production, Armidale, N.S.W. Australia

(+) The Agricultural Institute, Castleknock, Co. Dublin/Ireland

Efficiency in the beef industry is directly related to reproduction rate. Increases in fertility and calf survival will improve reproduction rate, but major changes are possible only through changes in fecundity.

Rapid initial increases in twinning rate may be achieved by intense selection of foundation stock, and performance records from several recently established herds confirm the expected phenotypic consequences of such selection. Continued selection within the line, based on dam's twinning record, should lead to annual rates of improvement of about $0.8 \mathrm{p}$. Ioo.

\title{
EINFLUSS DES GEBURTSVERLAUFES UND DES GEBURTSGEWICHTES DES KALBES AUF DIE FRUCHTBARKEIT BEIM SIMMENTALER FLECKVIEH
}

\section{GAILLARD}

\section{Schweizerischer Verband für künstliche Besamung, CH-Zollikofen/Schweiz}

Anhand von Geburtsinformationen und Angaben über die nachfolgende Besamungsperiode von ïber 40 ooo Simmentaler Fleckviehkühen wurde der Einfluss des Geburtsverlaufes und des Geburtsgewichtes des Kalbes auf die Fruchtbarkeit (Returnrate, Besamungsindex, Güstzeit) untersucht. Die Auswertungen wurden für Erstlingskühe und ältere Kühe getrennt durchgeführt.

I. Der Anteil der Kühe, die nach eines Schwergeburt wieder besamt werden, ist kleiner als nach Normalgeburten.

2. Die Fruchtbarkeit wird durch die Kaiserschnitte stark beeinträchtigt.

3. Die Schwergeburten haben nur bei den älteren Kühen einen schwach gesicherten negativen Einfluss auf die nachfolgende Fruchtbarkeitslage. Bei den Erstlingskühen sind die Differenzen zwischen Normal- und Schwergeburten klein und nicht signifikant.

4. Nach Normalgeburten konnte nur bei den älteren Kühen eine signifikante Verschlechterung der Fruchtbarkeit mit zunehmendem Geburtsgewicht des Kalbes festgestellt werden.

FRÉQUENCES GGNIQUES E'T DÉSÉQUILIBRES DE “IINKAGE 》 AUX LOCUS HaL, PHI ET 6-PGD DANS QUELQUES RACES PORCINES IFRANÇAISES (RÉSUL'TATS PRIIIIMINAIRES)

\section{G. GUÉRIN, I. OLIIVIER et P. SEILIFR}

Département de Génétique animale, I.N.R.A., 78350 Jouy-en-Josas (France)

Un échantillon de 439 porcs appartenant aux races Landrace belge, Landrace français et Large White a été soumis à un test d'anesthésie à l'halothane. Des prélèvements sanguins nous ont permis de déterminer le génotype de 356 animaux aux locus de la phosphohexose isomérase (PHI) et de la 6-phosphogluconate déshydrogénase 6-PGD. Les fréquences de la sensibilité à l'halothane et les fréquences géniques aux trois locus Hal, PHI et 6-PGD ont été estimées, ainsi que les déséquilibres de "linkage "(D) entre ces trois locus pris deux à deux.

Les valeurs de D trouvées entre $\mathrm{Hal}$ et PHI confirment de nombreux résultats antérieurs montrant l'association préférentielle de $\mathrm{PH}^{\mathrm{B}}$ avec le gène de sensibilité à l'halothane qui serait le résultat d'une sélection en faveur de ce gène. Par contre, les déséquilibres significatifs observés en Landrace framçais entre $\mathrm{Hal}$ et 6-PGD et entre 6-PGD et PHI peuvent difficilement s'expljquer par la sélection et ils résulteraient plutôt de mélanges de populations, hypothèse qui pourrait d'ailleurs aussi être appliquuée au déséquilibre entre Hal et PHI. 